\title{
Caribbean golden orbweaving spiders maintain gene flow with North America
}

\author{
*Klemen Čandek ${ }^{1,6}$, Ingi Agnarsson ${ }^{2,4}$, Greta J. Binford ${ }^{3}$, Matjaž Kuntner ${ }^{1,4,5,6}$ \\ ${ }^{1}$ Evolutionary Zoology Laboratory, Department of Organisms and Ecosystems Research, National \\ Institute of Biology, Ljubljana, Slovenia \\ ${ }^{2}$ Department of Biology, University of Vermont, Burlington, VT, USA \\ ${ }^{3}$ Department of Biology, Lewis and Clark College, Portland, OR, USA \\ ${ }^{4}$ Department of Entomology, National Museum of Natural History, Smithsonian Institution, \\ Washington D.C., USA \\ ${ }^{5}$ College of Life Sciences, Hubei University, Wuhan, Hubei, China \\ ${ }^{6}$ Evolutionary Zoology Laboratory, Institute of Biology, Research Centre of the Slovenian Academy \\ of the Sciences and Arts, Ljubljana, Slovenia \\ *Correspondence to klemen.candek@nib.si
}

\begin{abstract}
The Caribbean archipelago offers one of the best natural arenas for testing biogeographic hypotheses. The intermediate dispersal model of biogeography (IDM) predicts variation in species richness among lineages on islands to relate to their dispersal potential. To test this model, one would need background knowledge of dispersal potential of lineages, which has been problematic as evidenced by our prior biogeographic work on the Caribbean tetragnathid spiders. In order to investigate the biogeographic imprint of an excellent disperser, we study the American Trichonephila, a nephilid genus that contains globally distributed species known to overcome long, overwater distances. Our results reveal that the American T. clavipes shows a phylogenetic and population genetic structure consistent with a single species over the Caribbean, but not over the entire Americas. Haplotype network suggests that populations maintain lively gene flow between the Caribbean and North America. Combined with prior evidence from spider genera of different dispersal ability, these patterns coming from an excellent disperser (Trichonephila) that is species poor and of a relatively homogenous genetic structure, support the IDM predictions.
\end{abstract}

Keywords: dispersal potential, intermediate dispersal model of biogeography, ballooning, Trichonephila, Nephila

\section{Introduction}

Among the archipelagos, the Caribbean offers one of the best researched natural arenas for addressing biogeographic hypotheses (Ricklefs and Bermingham 2008). Caribbean islands are numerous and are of sufficiently varied ages and sizes to provide an historical context that generated interesting biogeographic histories of the organisms that inhabit them. An emerging issue that is relevant to organismal biology, lineage diversification, as well as biogeographic histories and patterns, is the degree to which variation in dispersal propensity can predict species richness of lineages (Laube et al. 2013; Borda-de-Água et al. 2017). The Caribbean is an ideal archipelago to pose these questions. 
The recently reformulated Intermediate Dispersal Model of biogeography (hereforth IDM) (Claramunt et al. 2012; Agnarsson et al. 2014) posits that differences among comparable lineages in dispersal potential over long distances affects their levels of gene flow over discrete units, such as islands, and that this variation is reflected in species richness patterns among these lineages. For example, if lineages contain poor dispersers, these organisms rarely colonize remote islands, leading to overall low species richness. Conversely, those lineages that are biologically capable of long distance travel may maintain such a lively gene flow among islands, or between island and continent, as to severely restrict speciation. Finally, those organisms with intermediate dispersal potential get to be carried to remote island rarely enough so that their founding populations may start to speciate, a hypothetical scenario that may result in the highest species richness. What this model implies is that biological attributes that define higher taxa, say genera, may link to the overall potential how these organisms disperse, and therefore affect their species richness, and biogeography.

The IDM, therefore, predicts species variation in richness among lineages to be a consequence of varying dispersal potential. However, in order to test the general validity of the model, one needs to identify appropriate test lineages. Ideally, these would be codistributed in an archipelago, be of comparable taxonomic ranks, and furthermore exhibit a measurable variation in phenotypes that pertain to dispersal. This has rarely been done, as studies testing the IDM have mainly focused on either only excellent dispersers (Claramunt et al. 2012) or solely poor dispersers (Pabijan et al. 2012), on lab reared organisms (Venail et al. 2008), or they included multiple lineages of incomparable taxonomic ranks (Agnarsson et al. 2014). In this vein, our prior work has compared a tetragnathid spider lineage with a hypothetical low dispersal potential (Cyrtognatha) (Čandek et al. 2018a) with its close relative (Tetragnatha) (Čandek et al. 2018b) over the Caribbean and the mainland. We found Tetragnatha to be extremely species rich in the Caribbean and attributed this richness to a biology that has elements of excellent dispersal, mixed with repeated secondary loss of dispersal ability, all this resulting in a mixed pattern of cosmopolitan, as well as narrowly endemic lineages. In comparison, Cyrtognatha was relatively species poor, with exclusively single island endemic species. We here explore another lineage that we a priori expect to show excellent dispersal ability that is co-distributed over the Caribbean.

Our present study thus focuses on the nephilid genus Trichonephila Dahl in the Caribbean. Trichonephila is a global genus of golden orbweavers (Nephilidae) that contains species known to readily cross long, overwater distances (Kuntner et al. 2018). Only two species are known in the New World, with T. clavipes distributed widely from North to South America (Kuntner 2017). We aim to test this single species status in Americas through a Caribbean transect, and with numerous terminals from other parts of the New World. We predict that in accordance with the IDM, Trichonephila will be species poor compared with the above tetragnathids, and will show the least structured genetic pattern over the archipelago. If so, this would implicate a lively gene flow over all the islands.

\section{Results and Discussion}

Our analyses reveal a phylogenetic and population genetic structure of $T$. clavipes consistent with a single species over the Caribbean. While T. clavipes is monophyletic, species delimitation analyses detect more than a single species over the Americas. Using the best fit model, GMYC delineates two species (marked as A and B in Fig. 1). The vertical bar in Figure 1 indicates the threshold where GMYC model shifts from phylogenetic Yule processes to coalescent population processes. Species A contains a dichotomy with a 
subclade containing the Caribbean and mainland North American populations, and a subclade with Colombian and Costa Rican populations. Species B is native to South and Central America. While species delimitations using some GMYC and a ABGD agree with the two species, alternatives exist (see Supplementary Figs. S1-S14). More precisely, some GMYC, mPTP and some ABGD suggest more than two species. However, these alternatives are less credible because they also suggest unrealistic species lumps of morphologically welldiagnosed species on other continents, such as T. komaci and T. sumptuosa being detected as a single species. Our conclusion that $T$. clavipes may in fact contain more than a single, Panamerican species, contradicts the classical morphological taxonomy (Kuntner 2017) but is consistent with a population genetic study in South America (Bartoleti et al. 2018).

The haplotype network (Fig. 2) depicts a single, well-represented haplotype present on the sampled Caribbean islands, as well as in continental North America. A few point mutations separate this highly frequent haplotype with those present on Jamaica, Mexico, Puerto Rico, and Turks and Caicos. Other haplotypes are more distant, and form two distinct groups, one in Colombia and Costa Rica (putatively conspecific with the Caribbean), and another in Brazil, Panama and French Guiana that corresponds to the above species B. This haplotype network is consistent with two or three species of $T$. clavipes. It also strongly suggests that the Caribbean populations maintain gene flow with the North American mainland.

These data reinforce the known biology of Trichonephila species as excellent dispersers. Although ballooning has not been directly observed in Trichonephila, it has been shown in the closely related and similar Nephila (Lee et al. 2015). Ballooning is an effective means of overcoming oceanic and continental barriers to gene flow, as several studies on Asian Nephila pilipes show (Lee et al. 2004, 2015; Su et al. 2006). More close relatives of $T$. clavipes also show extremely wide, genetically poorly structured population patterns worldwide. Good examples are $T$. inaurata that maintains gene flow between Africa and the islands of the Western Indian Ocean (Kuntner and Agnarsson 2011), as well as T. edulis and T. plumipes reported to travel seasonally from Australia to New Zealand (Harvey et al. 2007). There also seems to be a constant gene flow between the Korean and the Japanese populations of T. clavata (Jung et al. 2006).

Trichonephila clavipes resembles the Caribbean pattern detected in the araneid Argiope argentata where island populations clearly interbreed (Agnarsson et al. 2016). At a higher taxonomic level and within the area of interest, the Caribbean Trichonephila contrasts the two tetragnathid lineages: Cyrtognatha is a relatively poor to intermediate disperser with significant species richness and high endemism, Tetragnatha is a dynamic disperser lineage, with species apparently ranging from extremely good to relatively poor, and shows a high species richness and a mixed endemic to widespread mix of species. Trichonephila is an excellent disperser with a single species over the archipelago, exhibiting little genetic structure. Although Tetragnatha is an unusual case with apparently frequent evolutionary chance in dispersal potential, this triplet of genera provides preliminary support of the IDM.

In conclusion, $T$. clavipes does not seem to represent a single species over the Americas. The nominal T. clavipes is from the Caribbean (type is from Jamaica), but this species also inhabits North America, as well as parts of Central and South America. Over this vast geographical area, $T$. clavipes populations maintain a lively gene flow, suggesting that these spiders undertake airborne travel over the archipelago.

\section{Methods}




\section{Dataset assembly}

Our total dataset contains every available $T$. clavipes cytochrome c oxidase subunit 1 (COI) sequence from the combined Caribbean + USA region $(\mathrm{N}=58)$, an equal number of COI sequences randomly selected from Brazilian T. clavipes (Bartoleti et al. 2018), and every available sequence of $T$. clavipes from other areas (4 x Panama, 4 x Colombia, 1 x French Guiana, 1 x Costa Rica, 1 x Mexico). We also targeted other Trichonephila global exemplars (1 to 6 terminals per species, 8 species total), and four individuals of Nephila pilipes as the outgroup (Supplementary table S1). We emphasized the richness in geographic terminal coverage over that of using more genes with fewer terminals. Using COI marker to resolve phylogeographic questions is a common, and valid approach (Su et al. 2006; Čandek and Kuntner 2015; Bartoleti et al. 2018).

\section{Phylogenetic reconstructions}

To obtain ultrametric phylogenies, we performed Stepping Stone sampling and Bayes Factor test (Baele et al. 2012) within BEAST2 (Bouckaert et al. 2014). We reduced the total dataset (Supplementary table S1) and constrained the topology according to a phylogenomic hypothesis (Kuntner et al. 2018). Model tests (Supplementary note S2) selected a strict clock with the rate 0.0112 (Bidegaray-Batista and Arnedo 2011) and a coalescent constant population tree prior. We used bModelTest (Bouckaert and Drummond 2017) as nucleotide substitution model.

We reconstructed a Bayesian phylogeny using the reduced dataset as above in MrBayes (Huelsenbeck and Ronquist 2001), running four independent MCMC chains with 30 million generations, $25 \%$ burn-in and a sampling frequency of every 1000. GTR+G+I was the nucleotide substitution model (Darriba et al. 2012). Finally, we ran another Bayesian phylogeny for the T. clavipes ingroup only, with settings as above, except with 10 million generations.

\section{Species delimitations}

For species delimitation analyses, we employed three methods, the Generalized Mixed Yule Coalescent (GMYC) (Fujisawa and Barraclough 2013), the Multi-rate Poisson Tree Processes (mPTP) (Kapli et al. 2017) and the Automatic Barcode Gap Discovery (ABGD) (Puillandre et al. 2012). We ran GMYC delimitations in "splits" package of R version 3.5.1. (R Core Team 2018), using the utrametric tree and testing single, as well as multiple thresholds settings. We ran mPTP delimitations online using default settings and with ultrametric as well as Bayesian trees. For ABGD delimitations, we uploaded fasta sequences to its online platform and tested all three implemented substitution models (JC69, K80 and Simple distance). Here, we present a GMYC delimitation result using the best model for the data (Fig. 1) while 14 additional delimitation results are in the supplementary material (Supplementary Figs. S1 to S14).

\section{Haplotype network reconstruction}

We used "pegas" package in R to reconstruct a haplotype network of all 127 T. clavipes individuals from 12 areas. We trimmed the sequences to equal lengths, resulting in 537 remaining nucleotides. The sizes of circles in the reconstructed network correspond to the frequency of a specific haplotype.

\section{References}


Agnarsson, I., R.-C. Cheng, and M. Kuntner. 2014. A multi-clade test supports the intermediate dispersal model of biogeography. PLoS One 9:e86780.

Agnarsson, I., S. M. LeQuier, M. Kuntner, R.-C. Cheng, J. A. Coddington, and G. J. Binford. 2016. Phylogeography of a good Caribbean disperser: Argiope argentata (Araneae, Araneidae) and a new 'cryptic' species from Cuba. Zookeys 2016:25-44.

Baele, G., P. Lemey, T. Bedford, A. Rambaut, M. A. Suchard, and A. V. Alekseyenko. 2012. Improving the accuracy of demographic and molecular clock model comparison while accommodating phylogenetic uncertainty. Mol. Biol. Evol. 29:2157-2167.

Bartoleti, L. F. de M., E. A. Peres, F. von H. M. Fontes, M. J. da Silva, and V. N. Solferini. 2018. Phylogeography of the widespread spider Nephila clavipes (Araneae: Araneidae) in South America indicates geologically and climatically driven lineage diversification. J. Biogeogr. 45:1246-1260.

Bidegaray-Batista, L., and M. A. Arnedo. 2011. Gone with the plate: The opening of the Western Mediterranean basin drove the diversification of ground-dweller spiders. BMC Evol. Biol. 11:317.

Borda-de-Água, L., R. J. Whittaker, P. Cardoso, F. Rigal, A. M. C. C. Santos, I. R. Amorim, A. Parmakelis, K. A. Triantis, H. M. Pereira, and P. A. V. Borges. 2017. Dispersal ability determines the scaling properties of species abundance distributions: a case study using arthropods from the Azores. Sci. Rep. 7:3899. Nature Publishing Group.

Bouckaert, R., and A. J. Drummond. 2017. bModelTest: Bayesian phylogenetic site model averaging and model comparison. BMC Evol. Biol. 17:42. BioMed Central.

Bouckaert, R., J. Heled, D. Kühnert, T. Vaughan, C.-H. Wu, D. Xie, M. A. Suchard, A. Rambaut, and A. J. Drummond. 2014. BEAST 2: A software platform for Bayesian evolutionary analysis. PLoS Comput. Biol. 10:e1003537.

Čandek, K., I. Agnarsson, G. J. Binford, and M. Kuntner. 2018a. Biogeography of the Caribbean Cyrtognatha spiders. Sci. Reports (in Rev.), doi: 10.1101/372979.

Čandek, K., I. Agnarsson, G. J. Binford, and M. Kuntner. 2018b. Global biogeography of Tetragnatha spiders reveals multiple colonization of the Caribbean. Mol. Phylogenetics Evol. (in Rev.)

Čandek, K., and M. Kuntner. 2015. DNA barcoding gap: Reliable species identification over morphological and geographical scales. Mol. Ecol. Resour. 15:268-277.

Claramunt, S., E. P. Derryberry, J. V. Remsen, and R. T. Brumfield. 2012. High dispersal ability inhibits speciation in a continental radiation of passerine birds. Proc. R. Soc. B Biol. Sci. 279:1567-1574.

Darriba, D., G. L. Taboada, R. Doallo, and D. Posada. 2012. jModelTest 2: more models, new heuristics and parallel computing. Nat. Methods 9:772-772.

Fujisawa, T., and T. G. Barraclough. 2013. Delimiting species using single-locus data and the Generalized Mixed Yule Coalescent approach: A revised method and evaluation on simulated data sets. Syst. Biol. 62:707-724. Oxford University Press.

Harvey, M. S., A. D. Austin, and M. Adams. 2007. The systematics and biology of the spider genus Nephila (Araneae: Nephilidae) in the Australasian region. Invertebr. Syst. 21:407.

Huelsenbeck, J. P., and F. Ronquist. 2001. MrBayes: Bayesian inference of phylogenetic trees. Bioinformatics 17:754-755.

Jung, J., J. Lee, J. Kim, and W. Kim. 2006. Genetic variations of the golden orb-web spider Nephila clavata (Araneae: Tetragnathidae) in Korea , using AFLP markers. Korean j.Genetics.

Kapli, P., S. Lutteropp, J. Zhang, K. Kobert, P. Pavlidis, A. Stamatakis, and T. Flouri. 2017. Multi-rate Poisson tree processes for single-locus species delimitation under maximum likelihood and Markov chain Monte Carlo. Bioinformatics 33:1630-1638.

Kuntner, M. 2017. Nephilidae. Pp. 191-192 in D. Ubick, P. Paquin, P. E. Cushing, and V. 
Roth, eds. Spiders of North America, an identification manual. American Arachnological Society.

Kuntner, M., and I. Agnarsson. 2011. Phylogeography of a successful aerial disperser: The golden orb spider Nephila on Indian Ocean islands. BMC Evol. Biol. 11:119. BioMed Central.

Kuntner, M., C. A. Hamilton, R.-C. Cheng, M. Gregorič, N. Lupše, T. Lokovšek, E. Lemmon, A. Lemmon, I. Agnarsson, J. A. Coddington, and J. E. Bond. 2018. Golden orbweavers ignore biological rules: Phylogenomic and comparative analyses unravel a complex evolution of sexual size dimorphism. bioRxiv, doi: 10.1101/368233.

Laube, I., C. H. Graham, and K. Böhning-Gaese. 2013. Intra-generic species richness and dispersal ability interact to determine geographic ranges of birds. Glob. Ecol. Biogeogr. 22:223-232. Wiley/Blackwell (10.1111).

Lee, J. W., L. Jiang, Y. C. Su, and I.-M. Tso. 2004. Is central mountain range a geographic barrier to the giant wood spider Nephila pilipes (Araneae: Tetragnathidae) in Taiwan? a population genetic approach. Zool. Stud. 43:112-122.

Lee, V. M. J., M. Kuntner, and D. Li. 2015. Ballooning behavior in the golden orbweb spider Nephila pilipes (Araneae: Nephilidae). Front. Ecol. Evol. 3:2. Frontiers.

Pabijan, M., K. C. Wollenberg, and M. Vences. 2012. Small body size increases the regional differentiation of populations of tropical mantellid frogs (Anura: Mantellidae). J. Evol. Biol. 25:2310-2324.

Puillandre, N., A. Lambert, S. Brouillet, and G. Achaz. 2012. ABGD, Automatic Barcode Gap Discovery for primary species delimitation. Mol. Ecol. 21:1864-1877.

R Core Team. 2018. R: A language and environment for statistical computing. R Foundation for Statistical Computing, Vienna, Austria.

Ricklefs, R. E., and E. Bermingham. 2008. The West Indies as a laboratory of biogeography and evolution. Philos. Trans. R. Soc. B Biol. Sci. 363:2393-2413.

Su, Y. C., Y.-H. Chang, S.-C. Lee, and I.-M. Tso. 2006. Phylogeography of the giant wood spider (Nephila pilipes, Araneae) from Asian-Australian regions. J. Biogeogr. 34:177191.

Venail, P. A., R. C. MacLean, T. Bouvier, M. A. Brockhurst, M. E. Hochberg, and N. Mouquet. 2008. Diversity and productivity peak at intermediate dispersal rate in evolving metacommunities. Nature 452:210-214.

\section{Data accessibility}

All data and protocols needed to replicate this study are included in the paper and its Supplementary Material.

\section{Contributions}

Research design, material acquisition, molecular procedures: All authors. Data analyses: K.Č. K.C. . and M.K. wrote the first draft of the paper. All authors contributed to writing and revising the paper.

\section{Competing Interests}

The authors declare no competing interests.

\section{Funding}

This work was supported by grants from the National Science Foundation (DEB-1314749, DEB-1050253), and the Slovenian Research Agency (J1-6729, P1-0236, BI-US/17-18-011). 


\section{Acknowledgements}

We thank the entire CarBio team (http://www.islandbiogeography.org/participants.html) for collecting the material across the Caribbean. Moreover, we thank Lisa Chamberland and other members of the Agnarsson lab (http://www.theridiidae.com/lab-members.html) for the help with material sorting and molecular procedures.

\section{Figure legends}

Figure 1: GMYC species delimitation splits T. clavipes into two species. Species (A) shows pronounced population structure with Caribbean + North America subclade and Colombia + Costa Rica subclade. Species B is South and Central American. Vertical bar represents a likely threshold for speciation processes in Trichonephila.

Figure 2: Haplotype network hints at two, or three species, of T. clavipes. Specimens from the Caribbean and North America form one well represented haplotype. Other, minor haplotypes are separated by very few mutations, suggesting high levels of gene flow in the area of interest. 


\section{Trichonephila clavipes}

Caribbean

+ North America

Colombia

+ Costa Rica

South America

+ Panama

\section{komaci}

sumptuosa

inaurata

senegalensis

clavata

fenestrata

sexpunctata

plumipes

N. pilipes 